\title{
Operating educational networks in Northern Ireland: the EQI shaped professional learning network
}

\author{
Joe O'Hara ${ }^{* 1}$, Patrick Shevlin ${ }^{1}$, Martin Brown ${ }^{1}$, and Gerry McNamara ${ }^{1}$ \\ ${ }^{1}$ Dublin City University, Institute of Education, Centre for Evaluation, Quality and Inspection, \\ Dublin, Ireland
}

\begin{abstract}
This paper reports on the operation of the DCU Shaped Professional Learning Network (DCU SPLN), an initiative of the Centre for Evaluation Quality and Inspection (EQI) at Dublin City University. The DCU SPLN was initially established in Belfast, Northern Ireland as part of the Erasmus+ Project, 'Polycentric inspections of networks of schools', the focus of which was to provide an assessment of the potential of polycentric inspection as a tool for improving school effectiveness and outcomes. The cluster has now grown to 140 schools organised in regional clusters throughout Northern Ireland. The paper provides examples of the impact of the network on policy and practice in Northern Ireland. Firstly, it has a Hearts and Minds driving force that is centered on the ethical use of firsthand evidence to drive school improvement and associated action research. Secondly, it is underpinned by the existence and support from external agencies, in this case, researchers at EQI. Thirdly, the professional development provided to schools by EQI and subsequent adjustments by participants and advisors had a direct ongoing positive impact on improving the actual quality of learning in individual schools. Finally, one of the key learning points was the gradual transfer of responsibility for professional learning provision from the EQI researchers to the leaders and teachers involved in the cluster. The paper concludes with drawing some general conclusions about the role of networks in educational practice.
\end{abstract}

Keywords: educational networks, Northern Ireland, shaped professional learning networks, polycentric evaluation.

\section{Introduction}

This paper presents the work undertaken by the Centre of Evaluation, Quality and Inspection (EQI), Dublin City University with school communities in Northern Ireland over the course of the past decade. This work emerged initially from research conducted as part of an EUfunded Erasmus+ project titled "Polycentric inspections of networks of schools", the focus of which was to provide an assessment of the potential of polycentric inspection as a tool for improving school effectiveness and outcomes $[1,2]$. In recent years, this engagement has expanded to involve schools in the creation of a dynamic, research focused, networked

* Corresponding author: joe.ohara@dcu.ie 
culture that allows them take ownership of a wide range of professional and organisational initiatives to enhance educational outcomes at single school and regional levels [3-9]. The structure developed to facilitate this culture has been named the "DCU Shaped Professional Learning Network (DCU SPLN)" and it now numbers 140 schools cooperating across a series of local clusters [10].

\section{Methods}

The DCU SPLN explores the use and potential of polycentric inspection as a mechanism for educational enhancement across four European countries. Within Northern Ireland, this work saw researchers engage with a wide range of school communities, NGO's and other key educational stakeholders in the community of West Belfast. The research also required a focus on existing structures of inspection and quality assurance and for this reason the establishment some years ago of "Area Learning Communities" (ALCs) that consist of clusters of post-primary schools (including special schools) was particularly relevant to the establishment and development of the DCU SPLN. In the next parts of this paper we will explain the key concepts of the conducted research, the core values and principles of school network organization established in Nothern Ireland and the outcomes of the DCU SPLN with regard to the type of innovative practices applied in this project.

\section{Results: school networks in Northern Ireland}

Currently, there are 30 ALCs in Northern Ireland, which are defined by the Department of Education as "voluntary coalitions of schools which can be an efficient base for planning and collaboration to meet the needs of students in an area and for focusing on quality and sharing best practice" [11]. A core value of the networks is their voluntary nature, a fact that becomes more significant when the nature of their extensive engagement with the schools inspectorate is considered and in particular the parallel development of the area-based inspection structure [11].

First developed in 2005, area inspections focus on particular aspects of education across different stages in a geographical area.

The aim of all inspections is to promote improvement, the goal of the area inspection is to assess the effectiveness, adequacy relevance, and appropriateness of the provision of training and education within a given geographical area, in preparing 14-19 year-old students to progress to further education, training or employment [12].

The following statement in the Chief Inspector's Report (2008-2010) shows the significance placed by the ETI on education organisations in the field of collaborative work to provide quality education [13].

It is essential that all organisations who work for the benefit of students continue to explore methods of working together to provide efficient transitions and a more coherent experience for all. The area-based assessments of transitions within two distinct areas emphasize the importance of well-informed self-evaluation and of making more connections through working with stakeholders in order to raise standards and to achieve better outcomes for learners.

Here then is a system where the concepts of networking for effectiveness and improved outcomes were embedded into the formal accountability structures from a very early stage and helped provide a focus for emerging area communities whilst also providing the structures for engagement and communication that were deemed essential by writers in the field [14-16]. 
In practice, the focus of area-based inspections varies. It can be specifically related to strategic planning for education and training within the area, the quality of learning for students and the efficiency of the transition arrangements for young people within and across the various sectors. At its centre is an understanding of the importance of assessing quality in a developmental manner across a region or area rather than in the discrete community of an individual school. For this reason much of what is explored addresses the nature of connections at area or community level and many of the quality judgments are communicated at this level also.

The impact of this can be seen in the reporting modality chosen and the manner in which a network or ALC is engaged with when outcomes are being communicated. In practice, when the inspection is complete, a report is provided to the ALC and an overall judgement is made detailing the main strengths and areas in need of improvement at this level [13]. Inspection judgements fall within a quality continuum ranging from outstanding to unsatisfactory (outstanding, very good, good, satisfactory, inadequate, and unsatisfactory). A set of quantitative terms is applied to describe the extent to which an organisation is achieving its objectives, namely, "Very few/a small number (less than $10 \%)$ ", "A minority $(10 \%-$ $29 \%)$ ", "A significant minority (30\%-49\%)", "A majority $(50 \%-74 \%)$ ", "Most $(75 \%-$ 90\%)", "Almost/nearly (more than 90\%)". The report contains quantitative comparative data on such areas as key stage assessment results for the area in comparison with the averages of Northern Ireland and the percentage of school leavers entering employment or higher or further education in the area. This type of data allows for engagement with network level outcomes and places the onus on the ALC or network to develop approaches to address gaps in provision while at the same time developing on those modes of action that have resulted in the enhancement of educational provision across the network.

It is from this rich hinterland of practice and innovation that the DCU SPLN emerged. It has at its centre an awareness of the need to connect school communities in a manner that allows them share practices while at the same time preserve their distinct cultures, values and histories.

\section{Discussion: outcomes of network engagement}

A very effective way of professional learning has emerged in the dynamic growth of the DCU SPLN, a mode of learning that the research team chose to characterise as a Shaped Network. On the one hand, those involved in the network could operate with a great deal of autonomy to choose their own focus for school improvement and associated school-based action research. On the other hand, they were also required to operate with a clearly defined focus using the school development plan as the foundations for the research and the rigorous use of first-hand evidence to measure impact.

The work of the DCU SPLN is reviewed regularly and a number of key findings have emerged. Firstly, it has a Hearts and Minds driving force that is centered on the ethical use of first-hand evidence to drive school improvement and associated action research. Secondly, it is underpinned by the existence and support from external agencies, in this case, researchers at EQI. All parties emphasised the central importance of regular contact with and follow-up by members of EQI. This was perceived as vital in growing the network and guided schools to focus on using available first-hand evidence including statistics to examine issues around teaching and learning and teacher professional learning related to the action research topic chosen in their individual schools.

One of the vital questions is whether it can be proved that activities aimed at improving cooperation and providing professional development opportunities for teachers do lead to improved learning for students. It must be noted that there was substantial agreement by participants in the DCU SPLN schools that the professional development provided during 
the study, particularly the ongoing discussions of best practice emerging, and subsequent adjustments by participants and advisors if needed, enabled the case study work to have a direct ongoing positive impact on improving the actual quality of learning in individual schools.

A key learning point that also emerged was the gradual transfer of responsibility for professional learning provision from the EQI researchers to the leaders and teachers involved in the network/cluster. The staff involved from the various schools reported that because they were able to take the advice given and contextualize the advice given to their school situations, including a strong link with their school development plans, this empowered not just the staff attending the professional learning sessions but also those back in participants individual schools to take ownership of their own professional learning throughout the period of the action research. A key strategic point worth making here is that, if this model of professional learning was adopted more generally, then the appropriate role of professional development support becomes one of facilitation and enablement as opposed to any generic or disconnected support that might be offered to schools.

Finally, an important aspect of this research to be considered is how to take this successful network's results regarding the use of school-based action research to effect school improvement in other schools and other jurisdictions. The participants in the network would say, that the key is to convince teachers to use non-bureaucratic techniques to look at Firsthand Evidence of how good learning is in the classroom but to do this where possible and predominantly in a non-judgmental and collegiate manner. We call this horizontal selfevaluation. The work in these case studies suggests that there is also a place for vertical or judgmental self-evaluation, if we focus on the quality of learning, and not have a fixation on teaching performance. However, there is an ethical need to be clear as to which technique is being used for self- evaluation and more particularly for what purpose.

\section{Conclusion}

The outline of the origins, development and current configuration of the DCU SPLN is presented in order to provide a potential model for other networks of professional practice to consider. This network of school communities developed from a pre-existing school evaluation and quality assurance structure, was nurtured through the intervention of researchers interested in developing a polycentric mode of educational evaluation and came to fruition when school communities took control of the structure to allow them collaboratively enhance teaching and learning practices at individual school level. The DCU SPLN remains a dynamic, iterative and flexible entity drawing on a range of expertise and seeking to enhance the value of the professional knowledge and experience present within the school communities. In recent months the value of the existing network has been reinforced by its transition, in certain cases, to a support network for school leaders seeking to navigate their way through the challenges of the Covid-19 pandemic. While still anecdotal there is certain evidence to suggest that the DCU SPLN has become an important vehicle for school leaders to share best practice, offer emotional and professional support and initiate new modes of cooperation and communication.

\section{References}

1. F.J. Janssens, M.C. Ehren, Evaluation and Programme Planning, 56, 88-98 (2016). http://dx.doi.org/10.1016/j.evalprogplan.2016.03.012

2. J. O'Hara, M. Brown, G. McNamara, P. Shevlin, Journal of Educational

Research, 38(1), 33-52 (2019). https://doi.org/10.6018/rie.397201 
3. C. Azorín, A. Harris, M. Jones, School Leadership and Management, 40(2-3), 111-127 (2020). https://doi.org/10.1080/13632434.2019.1647418

4. C. Chapman, D. Muijs, School Effectiveness and School Improvement, 25(3), 351-393 (2014). https://doi.org/10.1080/09243453.2013.840319

5. A.J. Daly, K.S. Finnigan, Journal of School Leadership, 22(3), 493-530 (2012). https://doi.org/10.1177/105268461202200304

6. C. Hands, School Effectiveness and School Improvement, 21(2), 189-207 (2010). https://doi.org/10.1080/09243450903553993

7. A. Hargreaves, M. Fullan, Professional Capital: Transforming teaching in every school (Teachers College Press, New York, 2012).

8. R. Prenger, C.L. Poortman, A. Handelzalts, Journal of Teacher Education, 70(5), 441452 (2018). https://doi.org/10.1177/0022487117753574

9. S. Rincón-Gallardo, M. Fullan, Journal of Professional Capital and Community, 1(1), 5-22 (2016). https://doi.org/10.1108/jpcc-09-2015-0007

10. P. Shevlin, M. Brown, G. McNamara, J. O’Hara, Sharing the Impact of Shaped Professional Learning Networks in Challenging Times ((EQI) Centre for Evaluation, Quality and Inspection, Dublin, 2019)

11. Department of Education (DENI), Delivering the Entitlement Framework by 2013: Guidance for Schools on the Next Phase of Implementation (DENI, Bangor, 2010)

12. Education and Training Inspectorate (ETI), Report of A Pilot Area Inspection within the Greater Newry Area (Department of Education Northern Ireland, Bangor, 2005)

13. Education and Training Inspectorate (ETI), Chief Inspector's Report (Department of Education Northern Ireland, Bangor, 2010)

14. C. Chapman, Journal of Educational Administration, 57, 554-570 (2019).

15. C. Chapman, H. Chestnutt, N. Friel, S. Hall, K. Lowden, Journal of Professional Capital and Community, 1(3), 178-197 (2016). https://doi.org/10.1108/jpcc-03-20160007

16. K. Provan, P. Kenis, Journal of Public Administration Research and Theory, 18(2), 229-252 (2008). https://doi.org/10.1093/jopart/mum015 